\title{
BOUNDARY CONDITIONS APPLIED ON BEARING CORNER IN DIRECT ALUMINUM EXTRUSION
}

\author{
W.Assaad ${ }^{1 *}$ H.J.M. Geijselaers ${ }^{1}$, J. Huétink $^{1}$ \\ ${ }^{1}$ Faculty of Engineering Technology, University of Twente
}

\begin{abstract}
Finite element analysis in aluminum extrusion is faced by several problems such as number of degrees of freedom, calculation time, large deformation and flow conservation. The problem of large deformation is overcome by applying the Eulerian formulation. The problems concerning number of degrees of freedom, calculation time can be overcome by simplifying the model especially at the bearing corner. On the one hand, detailed modeling of the bearing corner will increase the complexity of the analysis. On the other hand, simplified modeling of the bearing corner will face problems such as locking of the bearing corner node and loss of flow conservation. A sharp corner and modified corner geometry are examples of the simplified modeling. Moreover, boundary conditions will be applied at the bearing corner node in order to solve the problem of its locking and to satisfy the flow conservation condition. These boundary conditions include specifying a normal or formulating a constraint equation. This paper focuses on the calculation of the normal or constraint equation that can be applied either at a sharp corner or after modifying the corner geometry. Different elements are checked in this study such as plane strain, axisymmetric and tetrahedron elements. Finally, the extrusion force and average exit velocity are investigated and compared with a reference model. In the reference model a round corner with $0.5 \mathrm{~mm}$ radius is built, contact boundary condition is applied between the die and aluminum, and Arbitrary Lagrangian Eulerian formulation is applied. The finite element analysis is performed in the in-house implicit finite element code "DiekA".
\end{abstract}

KEYWORDS: Extrusion, Eulerian, Flow conservation, Normal

\section{INTRODUCTION}

Aluminum extrusion process is not restricted to the production of simple profiles, but it is expanded to produce profiles that are used in industry. Research is concentrated on decreasing the amount of scrap by replacing the trialpressing with finite element simulations. In the analysis, stick boundary conditions are applied on the surfaces in contact with cylinder and die face, and fully slip boundary conditions are applied on the surfaces in contact with the bearing instead of contact boundary conditions. In the die geometry there are tiny entities that influence the out flow velocity and the extrusion force. One of these entities is the fillet at the bearing corner. Fine discretization of these entities will increase the number of degrees of freedom tremendously and coarse discretization of these entities will lead to problems such as locking of the bearing corner node or non conserved material flow. Therefore, studies on applying boundary conditions at the bearing corner node are required. In the current study, the normal or constraint equation boundary conditions are presented for sharp corner and modified corner geometry and compared with the triple node model [2] and a reference model.

*Faculty of Engineering Technology, University of Twente P.O.Box 217, 7500 AE Enschede, The Netherlands, phone: +31-(0)53-4894069, email: w.assaad@ctw.utwente.nl

\section{BOUNDARY CONDITIONS APPLIED TO THE BEARING CORNER NODE}

In literature several methods treat the problem of a flow around sharp edge such as a bearing corner in aluminum extrusion. These methods include construction of a normal from the flow field near the node, implemented by van Rens [1], triple node implemented by Lof [2] and average normal [3]. In van Rens' method the normal is calculated iteratively and the direction of the normal is not constant in the whole simulation. This method increases the calculation time and it is not clear whether the material flow is conserved or not. The other two methods depend on the mesh and they are applied in the preprocessing stage. In the triple node method three nodes are created at the bearing corner and their degrees of freedom are connected in such a way that the nodes will move around the corner as shown in figure 1. In this method the material flow is conserved but it consumes more time in preprocessing especially in 3D simulations and the extrusion force is underestimated because node $\mathrm{k}$ moves freely in the extrusion direction as shown in figure 1 . In the average normal method, the normal at the bearing corner node is calculated by averaging the normals of the outer surface areas of all the elements intersected at that node. The average normal method does not add time to the preprocessing and the extrusion force is acceptable but the material flow is not always conserved. Therefore, some methods are stud- 
ied such as specifying a normal at a sharp corner and applying boundary conditions at the corner after modifying its geometry. They are judged with respect to preprocessing time, material flow conservation and extrusion force.

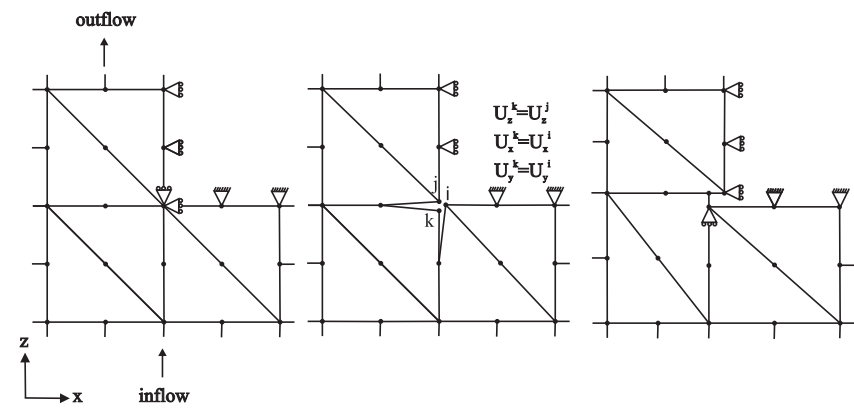

Figure 1: Triple node model [2]

\subsection{SPECIFYING A NORMAL TO A SHARP COR- NER}

Specifying a normal at a sharp corner can be performed in two ways. In one way, a local coordinate system at the corner node is specified, its movement in the direction perpendicular to the extrusion direction is suppressed and then the local coordinate system is rotated such that the movement of the node is suppressed in the direction of the normal. In the other way, a constraint equation between the velocity components of the corner node is formulated. The direction of the normal at the bearing corner as shown in figure 2 is calculated such that the net change in area (2D) or volume (3D) for elements connected to each corner node is zero. Then constraint displacement conditions are applied to the bearing corner node such that it moves perpendicular to that normal.
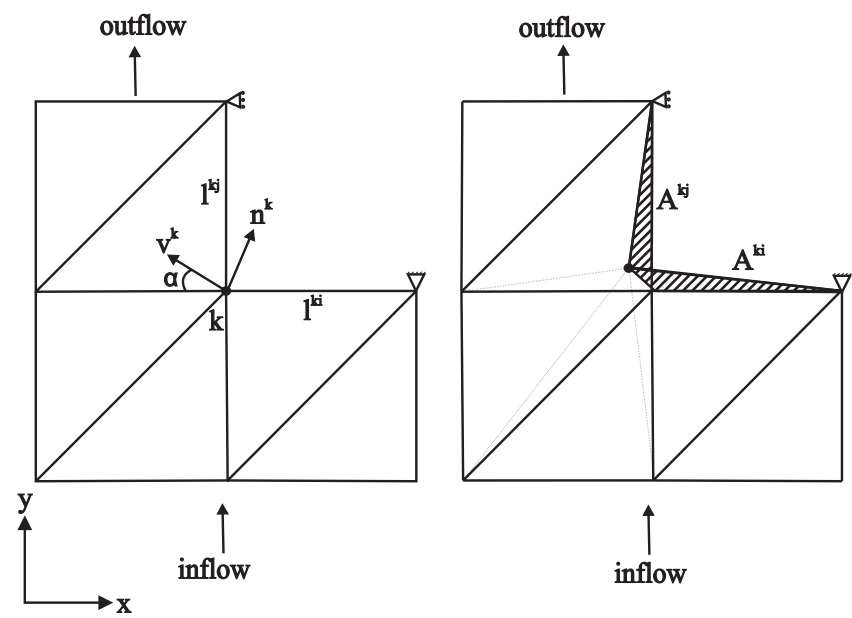

Figure 2: Normal

$$
A^{k i}=A^{k j}
$$

$$
\begin{gathered}
v^{k} \times l^{k i}=v^{k} \times l^{k j} \\
\tan \alpha=\frac{v_{y}^{k}}{v_{x}^{k}}
\end{gathered}
$$

where $v^{k}=$ velocity vector at the corner node, $v_{x}^{k}=$ $\mathrm{x}$-component of the velocity vector, $v_{y}^{k}=\mathrm{y}$-component of the velocity vector, $l^{k i}=$ size of element (i), $l^{k j}=$ size of element (j) $A^{k i}=$ area lost, $A^{k j}=$ area gained and $\tan \alpha=$ direction of the normal.

Equations 1,2 and 3 are relevant to the determination of the direction of the normal in plane strain simulations. But these equations don't hold for axisymmetric simulations because the distance between the center of gravity of each area to the axis of symmetry must be involved in the calculation. It is obvious from figure 2 that only the outer areas or volumes will influence the direction of the normal because the inner areas or volumes will cancel each other out. Therefore, the direction of the normal is mainly dependent on the size of the elements in the downstream $\left(l^{k i}\right)$ and in the upstream $\left(l^{k j}\right)$ regions of the bearing corner node. While in 3D simulations a different procedure is applied in calculating the direction of the normal. A node at the bearing corner is selected, a loop over all the elements intersected at this node is performed, a resultant vector for all face-area vectors is calculated and the direction of the normal is determined from the equation 4.

$$
\vec{A} \cdot \vec{v}=0
$$

where $\vec{A}=$ resultant vector for all face-area vectors, $\vec{v}=$ velocity vector of the corner node.

The extrusion process of a rod with an extrusion ratio 9 and a ram speed with $1 \mathrm{~mm} / \mathrm{sec}$ is discretized with ten node tetrahedron elements and analyzed isothermally with eulerian formulation. Concerning the boundary conditions, the nodes in the contact with the cylinder and the die face are sticking and the nodes in contact with bearing face are free in the extrusion direction. At the bearing corner the normal and the triple node methods are applied. The extrusion forces and out flow rates are predicted. In addition a $2 \mathrm{D}$ reference model with axisymmetric element is built and contact boundary conditions with 0.4 coefficient of friction are applied between the aluminum and the die. The results of the reference model are used in the evaluation of the normal and the triple method.

Figures 3 and 4 show that the flow rate criterion concerning the normal and triple node methods is satisfied but the extrusion forces differ in both methods. In the normal method the extrusion force exceeds that of the reference model by $3 \%$ because specifying a normal at the bearing corner node will resist its movement in the extrusion direction. In the triple node method the extrusion force is lower than that of the reference model by $9 \%$ because the bearing corner node moves freely in the extrusion direction. 


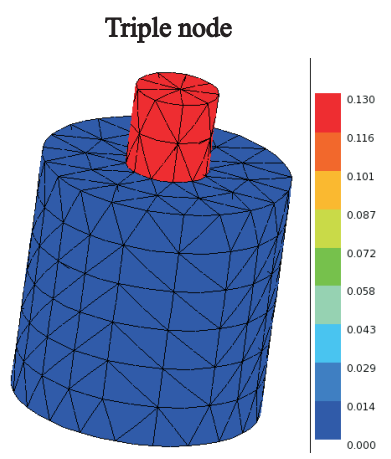

$\mathrm{F}_{\text {ext }}=0.15 \mathrm{MN}$ Qout $=9 \mathrm{~mm}^{3} / \mathrm{sec}$

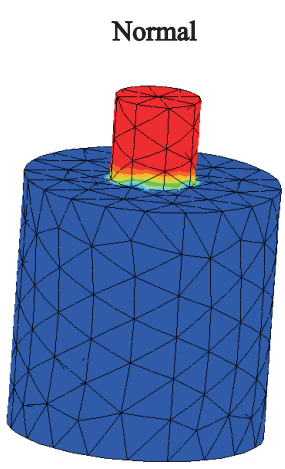

$\mathrm{F}_{\text {ext }}=0.17 \mathrm{MN}$

Qout $=9 \mathrm{~mm}^{3} / \mathrm{sec}$

Figure 3: Incremental displacement $[\mathrm{mm}]$ in the extrusion direction

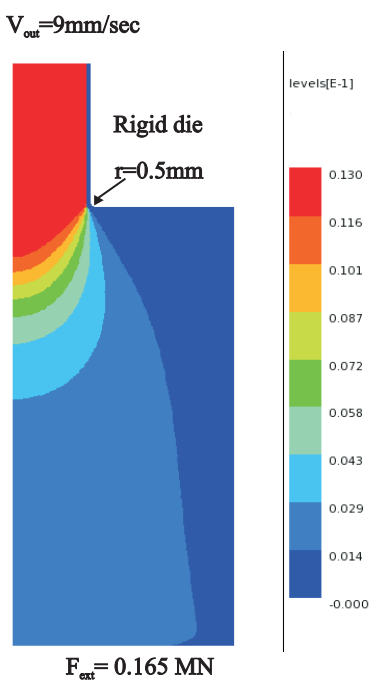

Figure 4: Incremental displacement [ $\mathrm{mm}]$ in the extrusion direction of the reference model $(d t=0.0014 \mathrm{sec})$

\subsection{BOUNDARY CONDITION APPLIED TO A CORNER AFTER MODIFYING ITS GEOME- TRY}

In the above method the influence of the fillet at the bearing corner is not considered. Therefore, its influence is regarded without increasing the number of degrees of freedom by changing the position of the bearing corner node.

$$
A^{1}+A^{2}=A^{3}
$$

where $A^{1}=\triangle \mathrm{PM} P^{1}, A^{2}=\triangle \mathrm{N} P^{1} N^{1}, A^{3}=\triangle P^{1} \mathrm{PN}$.

Discrete choices of the corner node are located in a square region with a side equal to half the element side. The position of the corner node is changed to different positions from $[i, j]$ to $[i+2, j+2]$ as shown in figure 5 . Similarly to the normal method the constraint equation is determined at each position of the corner node from equation 5 . The resulting constraint equation is non linear because of the formation of area $A^{2}$. Therefore, the equation is linearized by assuming the velocity at point $\mathrm{N}$ to be equal to the ram

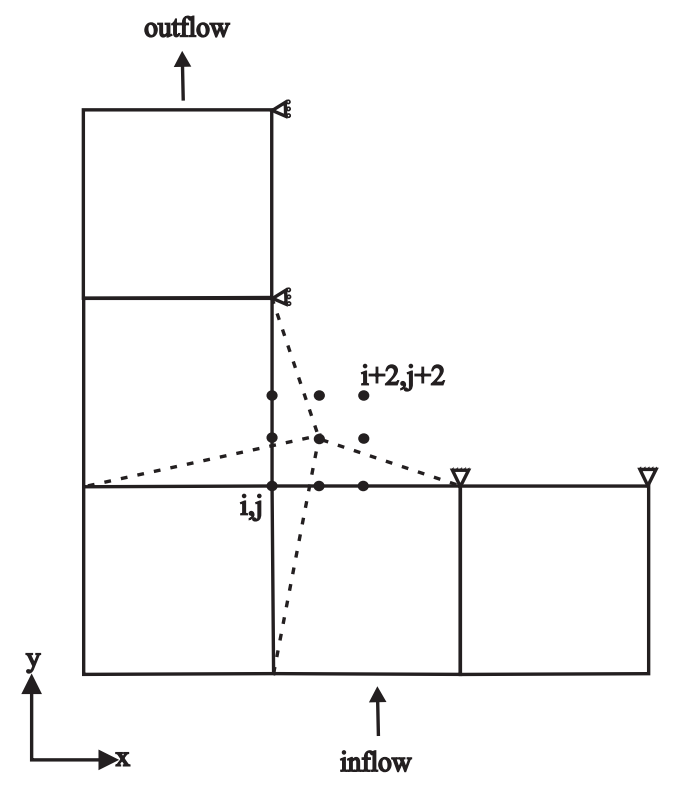

Figure 5: Choices of bearing corner node location

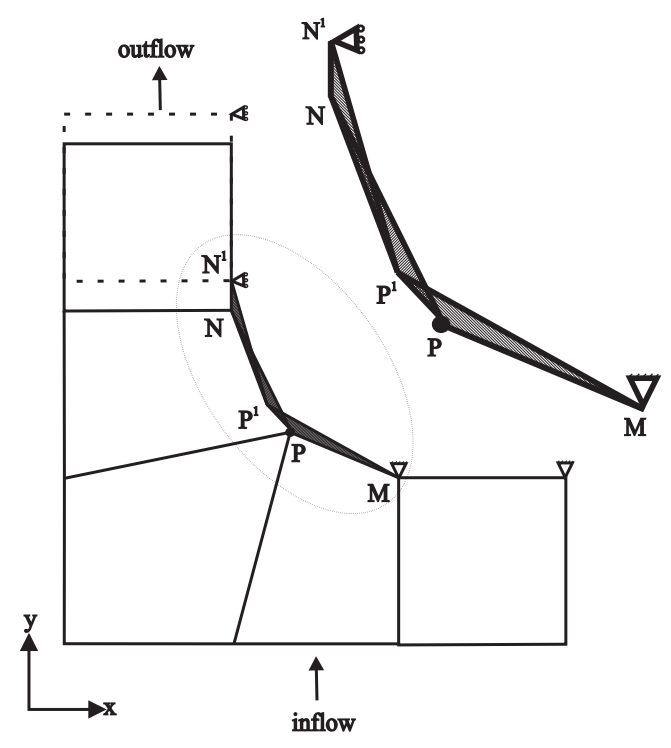

Figure 6: Formulation of constraint equation

speed times extrusion ratio. This study is applied in the simulation of an aluminum extrusion process with plane strain elements. At each position of the corner node the extrusion force and the flow conservation are checked and compared with respect to a reference model. The reference model is built similarly to the previous section unless the element type is plane strain.

Figure 7 shows unexpected results where the extrusion force increases as the corner bearing node moves radially. From figure 6 when corner node moves radially the area $A^{2}$ increases. Therefore the area $A^{3}$ must compensate the increase in $A^{2}$ by increasing the horizontal component of the velocity of the node. Figure 8 shows that the flow is non conservative at positions $[i+1, j],[i+2, j]$ and $[i+2, j+1]$ due to the linearized constraint equation. 


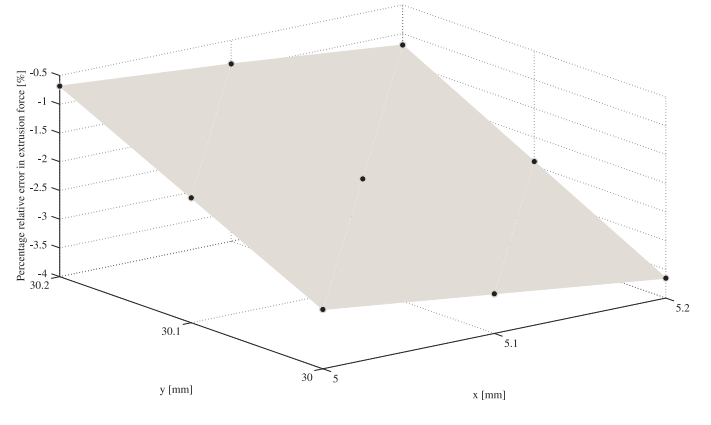

Figure 7: Absolute error in extrusion force with respect to reference

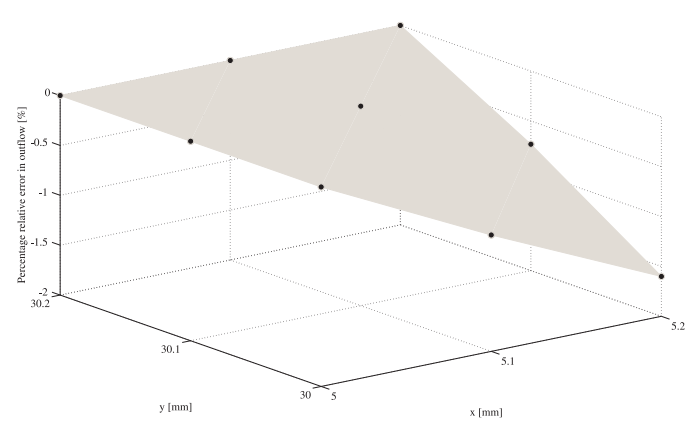

Figure 8: Error in out flow rate with respect to reference

\section{EXAMPLES}

\subsection{FLAT DIE}

Finally the extrusion of a $U$ shape profile is analyzed in order to show the applicability of the normal method in a real example. In the analysis the aluminum is discretized with ten node tetrahedron element. An isothermal calculation with a eulerian formulation is applied. Concerning the boundary conditions, the nodes in the contact with the cylinder and the die face are sticking and the nodes in contact with bearing are sliding free in the extrusion direction. Figure 9 shows the incremental displacement of the

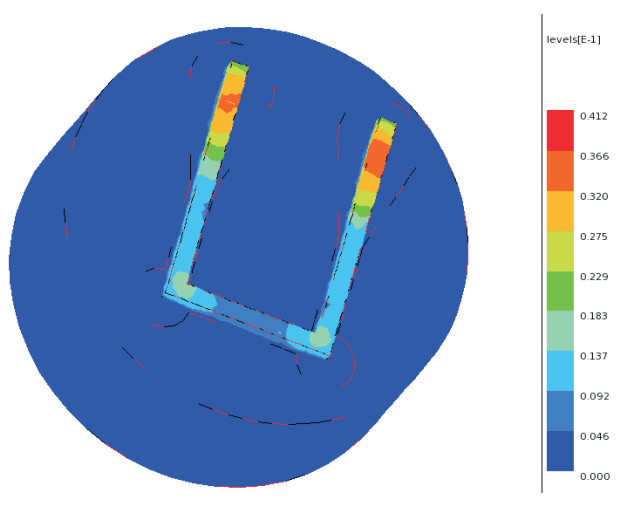

Figure 9: Incremental displacement $[\mathrm{mm}]$ in the extrusion direction $(d t=0.0001 \mathrm{sec})$

aluminum in the extrusion direction. The calculated out flow velocity is $9.954 \mathrm{~m} / \mathrm{min}$ and the experimental value is $10 \mathrm{~m} / \mathrm{min}$. Therefore, the percentage of relative error in out flow velocity is $0.46 \%$ which is an acceptable value.

\section{DISCUSSION}

Table 1 summarizes the calculated extrusion force and out flow velocity of the extrusion of the rod with axisymmetric and tetrahedron elements. In these calculations only the specified normal at the bearing corner is compared to the triple node model [2] and reference model. Table 1 shows a good agreement in the out flow velocity between the models and about $3 \%$ difference in the extrusion force between the specified normal model and the reference model. A slight increase in the extrusion force is more beneficial than waiting hours in preprocessing and solving the detailed model.

Table 1: Extrusion force and out flow velocity for the extrusion of a rod

\begin{tabular}{cccc}
\hline Model & Dimension & Extrusion force & Velocity \\
\hline Reference & 2 & $0.165 \mathrm{MN}$ & $9 \mathrm{~mm} / \mathrm{sec}$ \\
Triple node & 2 & $0.146 \mathrm{MN}$ & $9 \mathrm{~mm} / \mathrm{sec}$ \\
Triple node & 3 & $0.150 \mathrm{MN}$ & $9 \mathrm{~mm} / \mathrm{sec}$ \\
Specified normal & 2 & $0.152 \mathrm{MN}$ & $9 \mathrm{~mm} / \mathrm{sec}$ \\
Specified normal & 3 & $0.17 \mathrm{MN}$ & $9 \mathrm{~mm} / \mathrm{sec}$ \\
\hline
\end{tabular}

\section{CONCLUSION}

Finally, it is concluded that specifying the normal can overcome the problems such as preprocessing time and flow conservation. But modifying the corner geometry method seems to be unapplicable due to nonlinear constraint equation. The comparison between these methods is still limited because contact boundary conditions are not considered yet.

\section{ACKNOWLEDGEMENT}

This research was carried out under project number MC4.05221A in the framework of the Material innovation institute research programme in the Netherlands.

\section{REFERENCES}

[1] B.J.E. van Rens: Modelling friction near sharp edges using a Eulerian reference frame: application to aluminum extrusion, International Journal for Numerical Methods in Engineering (2002)

[2] Joeri Lof: Developments in finite element simulations of aluminum extrusion, pages 83-104 (2000)

[3] Koopman, A.J., Eindrapportage Simalex December 2006, University of Twente, December 2006 